\title{
Influência da tecnologia assistiva no desempenho funcional e na qualidade de vida de idosos comunitários frágeis: uma revisão bibliográfica
}

\author{
Influence of assistive technology on the functional performance and quality \\ of life of community-dwelling frail elderly people: a bibliographic review

\section{Resumo}

Dispositivos de tecnologia assistiva têm sido utilizados como forma de incrementar capacidade funcional, autonomia e qualidade de vida dos idosos fragilizados que vivem em seu domicílio. A fim de averiguar tal item, foi realizada revisão bibliográfica através de busca em bancos de dados, capítulos de livros, referências citadas nos artigos obtidos e artigos recebidos autores via internet ou correio. Os resultados obtidos indicaram diminuição da dependência dos idosos durante o cuidado pessoal, melhoria de sua socialização, incremento da tranquilidade e segurança dos cuidadores quanto à realização das tarefas funcionais pelos idosos e diminuição de episódios de reinternação e de gastos relacionados à saúde. Concluiu-se que os dispositivos de tecnologia assistiva são de grande valia para os idosos, aumentando

Palavras-chave: Equipamentos de Auto-Ajuda. Idoso Fragilizado.

Autonomia Pessoal. Qualidade de Vida. Assistência Domiciliar. Revisão.

Abstract

Assistive technology devices have been used to improve functional capability, autonomy, and quality of life of community-dwelling frail elderly people. In order to evaluate it, a bibliographic review was conducted through articles in databank, book chapters, other articles and by their authors who directly

\footnotetext{
Universidade Federal de Minas Gerais

Ciências da Saúde,Terapia Ocupacional, Fisioterapia Belo Horizonte, MG, Brasil

1 Departamento de Terapia Ocupacional da Universidade Presidente Antônio Carlos

2 Departamento de Fisioterapia da Universidade Federal de Minas Gerais
}

Correspondência / Correspondence

Valéria Souza de Andrade

E-mail: valeria-to@hotmail.com 
sent them by mail or e-mail. It was observed a decrease of those elderly people's dependence while taking care of themselves, improvement of their socialization, betterment of caregivers' safety and peacefulness regarding the performance of functional activities by elderly people, and a lowering of constant frail aging people's hospitalizations and concomitant health-related costs. The conclusion was that assistive technology devices are quite valuable to community-dwelling frail elderly people, improving their functional capacity, autonomy, and quality of life.
Key words: Self-Help Devices. Frail Elderly. Personal Autonomy. Quality of Life. Home Nursing. Review.

\section{INTRODUÇÃO}

Atualmente, admitem-se duas formas de envelhecimento: o usual ou comum e o bem-sucedido ou saudável. $\mathrm{Na}$ forma usual ou comum, os fatores extrínsecos, tais como tipo de dieta, sedentarismo e causas psicossociais, intensificariam os efeitos adversos que ocorrem com o passar dos anos, enquanto na forma de envelhecimento bemsucedido ou saudável esses fatores não estariam presentes ou seriam de pequena importância. Finalmente, há também os idosos frágeis, os quais apresentam modificações determinadas por afecções que tornam a pessoa idosa debilitada, dependente do auxílio de terceiros e, por vezes, extremamente difícil. ${ }^{1}$

Embora ainda não haja consenso quanto à sua definição, nos últimos 20 anos geriatras e gerontólogos vêm utilizando o termo fragilidade, no contexto do diagnóstico e do tratamento das manifestações de velhice patológica, mais especificamente para caracterizar os idosos mais debilitados e vulneráveis.

A sociedade brasileira é carente de programas de promoção, prevenção e recupera- ção da saúde do idoso. Consequentemente, o envelhecimento populacional brasileiro caracteriza-se pelo acúmulo de incapacidades progressivas nas suas atividades de vida diária, na medida em que estamos longe de atingirmos o envelhecimento bem-sucedido. A tendência é de se observar um número crescente de idosos que, muito embora destinados a viver cerca de 20 anos mais, encontram-se funcionalmente incapacitados e com uma saúde precária.

O crescimento demográfico dos idosos fragilizados no Brasil tem conduzido à demanda crescente de profissionais que utilizam abordagens diversas para intervir na progressão das doenças e suas comorbidades que frequentemente acometem essa população. Em países desenvolvidos, várias alternativas têm sido propostas, dentre elas, a tecnologia assistiva (TA) a qual se refere a diversos tipos de dispositivos de auxílios estratégicos que visam a reduzir o impacto da disfunção física, ao prover uma conexão entre as limitações funcionais do indivíduo e as demandas do meio físico. ${ }^{2}$ Vários tipos de TA têm sido propostos e implementados para atender aos idosos fragilizados e dependentes que têm necessidade de man- 
ter sua capacidade funcional, autonomia e qualidade de vida. No Brasil, entretanto, existe uma carência no conhecimento e aquisição de tais dispositivos, principalmente pelas classes sociais de baixa renda.

Diante disso, o objetivo deste trabalho foi buscar evidências na literatura, através de uma revisão bibliográfica, sobre o papel dos dispositivos de TA no aumento da capacidade funcional e na qualidade de vida dos indivíduos idosos fragilizados domiciliários.

\section{METODOLOGIA}

O estudo se constituiu em uma revisão bibliográfica cujos dados foram obtidos através de busca nos bancos da dados Pubmed (www.pubmed.gov.br), Bireme (www. bireme.br), Scielo (www.scielo.br), Portal Capes (www.periodicos.capes.gov.br) e OTSeeker (www.otseeker.com); capítulos de livros, referências citadas nos artigos obtidos, além de artigos recebidos dos próprios autores via internet ou correio. A busca se limitou a artigos entre 1980-2006 nos idiomas português e inglês.

$\mathrm{Na}$ busca bibliográfica foram obtidos 72 resultados, dentre os quais artigos científicos, capítulos de livros e teses de doutorado. Foram utilizados para a redação desta revisão bibliográfica 16 artigos científicos, devido à sua maior relevância para com o tema abordado. Dentre os artigos utilizados, observaram-se 11 estudos do tipo qualitativo, três estudos do tipo quantitativo e duas revisões narrativas (quadro 1).

Quadro 1 - Estudos utilizados na revisão bibliográfica

\begin{tabular}{|l|l|l|}
\hline Autor & Tipo de estudo / Amostra & \multicolumn{1}{c|}{ Resultados } \\
\hline $\begin{array}{l}\text { Agree E, } \\
\text { Freedman V. }\end{array}$ & $\begin{array}{l}\text { Qualitativo, longitudinal, } \\
\text { prospectivo, descritivo. }\end{array}$ & $\begin{array}{l}\text { - TA é usada para realizar AVDs e AVDIs. } \\
\text { - Indivíduos que usam apenas TA têm mais dificuldade } \\
\text { do que aqueles que recebem cuidado pessoal e usam } \\
\text { TA. }\end{array}$ \\
\hline $\begin{array}{l}\text { Agree E, } \\
\text { Freedman V. }{ }^{1}\end{array}$ & $\begin{array}{l}\text { Qualitativo, longitudinal, } \\
\text { descritivo. }\end{array}$ & $\begin{array}{l}\text { - TA é usada para aquisição de necessidades. } \\
\text { - Idosos usam TA quando recebem cuidado informal } \\
\text { ou formal. } \\
\text { - TA simples e barata pode levar à independência e } \\
\text { substituir necessidade de cuidadores formais. } \\
\text { - TA pode diminuir as demandas sobre o cuida dor. } \\
\text { TA leva à melhoria da qualidade de vida dos idosos e } \\
\text { de seus cuidadores. }\end{array}$ \\
\hline $\begin{array}{l}\text { O uso de TA prorroga necessidade de } \\
\text { institucionalização. }\end{array}$
\end{tabular}


Quadro 1 - Estudos utilizados na revisão bibliográfica (continuação)

\begin{tabular}{|c|c|c|}
\hline Autor & Tipo de estudo / Amostra & Resultados \\
\hline Chen T, et al. ${ }^{4}$ & $\begin{array}{l}\text { Qualitativo, longitudinal, } \\
\text { prospectivo, descritivo. } \\
20 \text { idosos fragilizados, } 64-87 \\
\text { anos. }\end{array}$ & $\begin{array}{l}\text { - Há necessidade de assistência física e verbal de } \\
\text { cuidadores. } \\
\text { - TA economiza tempo e energia, reduz frustração e } \\
\text { provê segurança. } \\
\text { - Há satisfação com uso de TA por parte de utilitário. } \\
\text { - A satisfação do utilitário da TA é reforçada por } \\
\text { cuidador. }\end{array}$ \\
\hline $\begin{array}{l}\text { Cornman J, } \\
\text { Freedman V, } \\
\text { Agree E. }{ }^{5}\end{array}$ & $\begin{array}{l}\text { Qualitativo, descritivo, } \\
\text { retrospectivo. } \\
45.810 \text { idosos fragilizados, } \\
65+\text { anos }\end{array}$ & $\begin{array}{l}\text { - Indivíduos que usam TA e não têm dificuldade com } \\
\text { tarefas diárias têm maior nível educacional. } \\
\text { - TA mais usadas pelos utilitários: bengala canadense, } \\
\text { dispositivos para transferência e cadeira de rodas. } \\
\text { - A TA é mais usada devido às mudanças nas condições } \\
\text { de saúde, ao não auxílio de terceiros ou ao interesse } \\
\text { na manutenção da independência. } \\
\end{array}$ \\
\hline $\begin{array}{l}\text { Freedman VA, et } \\
\text { al. }\end{array}$ & $\begin{array}{l}\text { Qualitativo,longitudinal, } \\
\text { descritivo. } \\
128.568 \text { indivíduos fragilizados, } \\
65+\text { anos. }\end{array}$ & $\begin{array}{l}\text { - Indivíduo realiza } 26 \% \text { das AVDs com o auxílio de } \\
\text { TA. } \\
\text { - Há diminuição do cuidado pessoal com uso de TA. } \\
\text { - Idoso recorre à TA quando não há cuidador. }\end{array}$ \\
\hline $\begin{array}{l}\text { Gitlin LN, } \\
\text { Luborsky MR, } \\
\text { Schemm RL. }\end{array}$ & $\begin{array}{l}\text { Qualitativo, longitudinal, } \\
\text { prospectivo. } \\
103 \text { indivíduos pós-AVE, } 55+ \\
\text { anos. }\end{array}$ & $\begin{array}{l}\text { - TA é positiva ou negativa dependendo se as } \\
\text { necessidades são ou não supridas. } \\
\text { - Indivíduo reconstrói rua auto -estima e bem estar caso } \\
\text { use TA. } \\
\text { - Comentários individuais capacitam médicos a } \\
\text { examinar seus pensamentos e sentimentos. }\end{array}$ \\
\hline $\begin{array}{l}\text { Gitlin LN, } \\
\text { Levine RE, } \\
\text { Geiger C? }\end{array}$ & $\begin{array}{l}\text { Quantitativo, experimental, } \\
\text { transversal, exploratório e } \\
\text { qualitativo, prospectivo. } \\
13 \text { idosos fragilizados, } 61-84 \\
\text { anos, descritivo. }\end{array}$ & $\begin{array}{l}\text { - TA é prescrita baseando -se no meio hospitalar aonde } \\
\text { indivíduo se encontra, em sua patologi a, em seus } \\
\text { próprios objetivos, nos valores culturais e } \\
\text { características físico-sociais de sua casa. }\end{array}$ \\
\hline $\begin{array}{l}\text { Gosman- } \\
\text { Hedström G, } \\
\text { Blomstrand C. }\end{array}$ & $\begin{array}{l}\text { Quantitativo, transversal, } \\
\text { descritivo, longitudinal. } \\
151 \text { idosos pós-AVE moderado, } \\
81 \text { anos (média). }\end{array}$ & $\begin{array}{l}\text { - Observou-se melhoria na execução de AVDs e na } \\
\text { qualidade de vida após uso de TA. } \\
\text { - A não melhoria da qualidade de vida com uso de TA } \\
\text { deve-se à inabilidade de realizar atividades. } \\
\text { - TA repercute positiva (e.x., auxilia na execução das } \\
\text { atividades) ou negativamente (e.x., relembra } \\
\text { indivíduo da incapacidade). }\end{array}$ \\
\hline
\end{tabular}




\section{Quadro 1 - Estudos utilizados na revisão bibliográfica (continuação)}

\begin{tabular}{|c|c|c|}
\hline Autor & Tipo de estudo / Amostra & Resultados \\
\hline $\begin{array}{l}\text { Gosman- } \\
\text { Hedström G, et } \\
\text { al. }{ }^{10}\end{array}$ & $\begin{array}{l}\text { Qualitativo, longitudinal, } \\
\text { prospectivo. } \\
249 \text { indivíduos pós-AVE. }\end{array}$ & $\begin{array}{l}\text { - A utilização de TA não é diferente de sua não } \\
\text { utilização. } \\
\text { - A maioria dos dispositivos foi usada ocasionalmente } \\
\text { ou sempre. } \\
\text { - Indivíduos preferiam realizar atividades sem TA. } \\
\text { - Dificuldade do uso de TA: disfunção cognitiva, não } \\
\text { desejo de uso e razões culturais/tradicionais. } \\
\text { - Vantagem de TA: prescrição para auxiliar no } \\
\text { desempenho. } \\
\text { - Desvantagem de TA: prescrição indiscriminada e com } \\
\text { pouco efeito para o indivíduo. }\end{array}$ \\
\hline Mann WC, et al. ${ }^{14}$ & $\begin{array}{l}\text { Qualitativo,prospectivo, } \\
\text { longitudinal. } \\
1.016 \text { idosos fragilizados } 75 \\
\text { anos (média). }\end{array}$ & $\begin{array}{l}\text { - TA mais importantes sob o ponto de vista dos } \\
\text { utilitários: lupa para aumento visual, bengala, cadeira } \\
\text { de rodas, andador e barra de apoio. } \\
\text { - Idosos fragilizados percebem TA como importante na } \\
\text { manutenção da independência físico-funcional. }\end{array}$ \\
\hline Mann WC, et al. ${ }^{13}$ & $\begin{array}{l}\text { Quantitativo,prospectivo, } \\
\text { transversal,exploratório. } \\
104 \text { indivíduos fragilizados, } 73 \\
\text { anos (média). }\end{array}$ & $\begin{array}{l}\text { - Grupo controle apresentou menor alteração } \\
\text { funcional (MIF) com uso de TA. } \\
\text { - Grupo experimental apresentou maior gasto } \\
\text { financeiro com internação e atendimento domiciliar. }\end{array}$ \\
\hline $\begin{array}{l}\text { Mello MAF, } \\
\text { Ramos LR. }{ }^{15}\end{array}$ & $\begin{array}{l}\text { Qualitativo,longitudinal, } \\
\text { descritivo, prospectivo. } \\
144 \text { idosos fragilizados, } 73-103 \\
\text { anos. }\end{array}$ & $\begin{array}{l}\text { - Setenta e três por cento dos indivíduos possuíam TA } \\
\text { devido à deficiência motora. } \\
\text { - TA foi prescrita por amigos, parentes, médicos, } \\
\text { fisioterapeutas e fonoaudiólogos. } \\
\text { - Quando TA é prescrita pelo amigo/familiar reforça } \\
\text { tendência à auto-medicação. } \\
\text { - Indivíduos não usam TA devid o ao preço ou por não } \\
\text { saberem do que se trata. } \\
\text { - Idosos que utilizam TA apresentam perdas motoras. } \\
\text { - Idoso que financia TA sugere negligência dos } \\
\text { profissionais de saúde. } \\
\text { - Quedas podem ser devidas ao déficit de profissional } \\
\text { que oriente modificações ambientais. }\end{array}$ \\
\hline Tomita M, et al..$^{18}$ & $\begin{array}{l}\text { Quantitativo, descritivo, } \\
\text { longitudinal, prospectivo. } \\
694 \text { idosos fragilizados, } 60+ \\
\text { anos. }\end{array}$ & $\begin{array}{l}\text { - Fatores que levam ao uso de TA: motivação, } \\
\text { gravidade e duração da doença, ingestão de } \\
\text { medicação, ausência de depressão, determinação em } \\
\text { viver independentemente, existência de suporte } \\
\text { social, recomendação do uso do dispositivo e } \\
\text { interação entre TA e ambiente de moradia. }\end{array}$ \\
\hline Wessels R, et al. ${ }^{19}$ & $\begin{array}{l}\text { Revisão narrativa } \\
\text {-x- }\end{array}$ & $\begin{array}{l}\text { - O não uso de TA pode ser devido à não aceitação da } \\
\text { incapacidade, à depressão, a produtos de má } \\
\text { qualidade, à barreira arquitetônica e à inapropriada } \\
\text { instrução em sua aquisição. }\end{array}$ \\
\hline
\end{tabular}




\section{RESULTADOS E DISCUSSÃO}

O objetivo principal da TA seria eliminar a lacuna entre o desempenho funcional de um indivíduo e as demandas das tarefas funcionais da vida diária, aumentando, consequentemente, a qualidade de vida. ${ }^{3} \mathrm{O}$ impacto do uso da TA no desempenho funcional ou na retenção das habilidades funcionais pré-adquiridas no maior nível possível, bem como a aquisição de independência e incremento da qualidade de vida, são comumente relatados. ${ }^{2,4,5,6,7}$ Também é relatada a economia no gasto de energia e de tempo para completar uma tarefa ${ }^{3,6}$ de atividades básicas de vida diária (ABVD), das atividades práticas de vida diária (AVDP) e das atividades instrumentais de vida diária (AIVD) ${ }^{4,8}$ quer seja dentro ${ }^{3,6}$ ou fora das residências. ${ }^{9,10}$

Vários aspectos físicos são melhorados com o uso da TA, dentre eles a prevenção ou a diminuição do risco de quedas e fraturas, ${ }^{2,8}$ a melhoria da mobilidade dentre os diversos cômodos da moradia e de acessibilidade ao meio ambiente, ${ }^{2}$ a redução de dor e do risco de lesões durante a execução das atividades de autocuidado, lazer ou trabalho ${ }^{2}$ e a minimização dos déficits relacionados às mudanças características do envelhecimento, tais como a diminuição da força muscular, da coordenação, do equilíbrio e a instalação (ou piora) de deficiência visual. ${ }^{2}$

O uso da TA tem também impacto no aspecto psicossocial, visto auxiliar o indivíduo na reconstrução do sentimento de auto- estima e bem-estar ${ }^{4}$ e ajudá-lo no caso de incapacidades no aprendizado, diminuindo ou eliminando a sua frustração para completar uma tarefa. ${ }^{3} \mathrm{O}$ constante uso da TA destaca-se na prevenção de futuras recorrências do sentimento de frustração devidas à incerteza e falta de confiança por parte do idoso e de seu cuidador, ${ }^{3,11}$ no fornecimento de sentimento de segurança ${ }^{3}$ e no encorajamento à independência do idoso fragilizado e melhoria de sua socialização. ${ }^{2,8}$ Tomita et al. ${ }^{5}$ destacam ainda que quando os idosos têm relações significativas para com outros indivíduos, sentem-se úteis e funcionalmente ativos. Caso isto ocorra, há condução automática a um estado psicossocial positivo, o qual propicia o uso de auxílios - dentre eles a TA -, que levam a um melhor convívio na comunidade e, portanto, ao incremento da função e ausência de estados depressivos. ${ }^{5}$

O uso dos dispositivos de TA é também identificado por auxiliar na diminuição de despesas financeiras do utilitário. Segundo a literatura pesquisada, a TA contribui para a diminuição de dependência durante o cuidado pessoal e o aumento de privacidade, para a redução dos gastos financeiros com cuidados providos em casa, ${ }^{3,8,12}$ para a substituição de cuidadores pelo uso da $\mathrm{TA}^{8} \mathrm{e}$ para diminuição de episódios de reinternação e concomitante decréscimo dos gastos financeiros desencadeados por problemas funcionais crônicos. ${ }^{8,12}$

Chen et al. ${ }^{3}$ observaram que os idosos têm mais satisfação ao utilizarem dispositi- 
vos de TA quando os cuidadores se mostram física ou verbalmente envolvidos. ${ }^{4} \mathrm{Tal}$ satisfação é corroborada por Mann et al., ${ }^{13}$ quando afirmam que os indivíduos idosos, devido à sensação de segurança, utilizam a TA visando a prevenir quedas, à manutenção do equilíbrio e à melhoria da mobilidade que aqueles dispositivos proporcionam. ${ }^{13}$

Além do idoso sob cuidado, o cuidador, pessoa que formal ou informalmente oferece auxílio para suprir uma ou mais dependências do indivíduo idoso à medida que elas se instalam, também pode beneficiar-se caso o aquele indivíduo se utilize da TA. O cuidador exerce papel muito importante ao reforçar a satisfação do idoso na utilização da TA, uma vez que se dedica ao ensino e auxilio físico da utilização daqueles dispositivos. ${ }^{3}$ Quando o idoso se utiliza de um dispositivo de TA para realizar suas tarefas, a segurança do cuidador é maior. ${ }^{14}$ Desta forma, o cuidador, submetido frequentemente ao stress crônico de cuidar do idoso, experiência diminuição de gasto de energia, melhora de qualidade de vida e consequente disponibilidade em ocupar-se em menor grau com o indivíduo sob seu cuidado quando este utiliza constantemente algum dispositivo de TA. ${ }^{8,11}$ Agree e Freedman ${ }^{8}$ salientam que os cuidadores informais observam que o uso da TA pode melhorar as habilidades funcionais dos idosos na comunidade, prorrogando desta forma a necessidade de um cuidador formal. ${ }^{8}$

Os autores pesquisados utilizaram vários dispositivos de TA para a condução de suas pesquisas. No tocante aos dispositivos mais utilizados, encontram-se aqueles relacionados às atividades que demandam mobilidade, como cadeira de rodas, andador e bengala, e dispositivos que facilitam a execução das ABVD, tais como barra de apoio, assento para aumento do vaso sanitário, banco para transferência da/para banheira, tapete antiderrapante, garra e esponja com cabos alongados, dispositivos para alimentação e vestuário, , ${ }^{8,10,13}$ auxílios para calçar sapatos e meias, faca arredondada, aparador para pratos e almofada para assento. ${ }^{4,13}$

A despeito dos benefícios observados com a utilização da TA, observou-se que há relutância por parte dos indivíduos idosos em utilizar tais dispositivos devido a motivos diversos. Gosman-Hedstön et al. ${ }^{11}$ observaram que idosos podem ter dificuldades em utilizar a TA por problemas relacionados à cognição ou ainda por razões culturais ou tradicionais. ${ }^{11}$ Outros motivos pelos quais idosos fragilizados podem não utilizar a TA são frustração ao terem que usar tais dispositivos para realizar as atividades, visto que os mesmos indicam suas limitações, desinteresse no aprendizado sobre como lidar com eles, inutilidade para suprir as necessidades, baixa qualidade estética, incômodo ao realizar as atividades com auxílio dos mesmos, má qualidade conduzindo ao estrago e consequentemente à dificuldade na manutenção deficitária, instrução e/ou prescrição por parte do profissional quanto à sua utilização e desconhecimento das vantagens, por parte do idoso., 2,15,16,17 Também cooperam para a não- 
utilização da TA por parte dos idosos o sentimento de vergonha, a negação da incapacidade ${ }^{2,4,17}$ e a existência de barreiras físicas no meio ambiente onde o idoso reside. ${ }^{9,16,17}$

Segundo Gosman-Hedström et al., ${ }^{11}$ indivíduos residentes em países que dão suporte à utilização de dispositivos de TA comumente os recebem de forma gratuita nos centros de saúde ou são reembolsados pelas políticas de saúde após adquiri-los. ${ }^{11}$ Tal conduta é louvável em locais onde a população idosa tem crescido, sendo pertinente concluir que os sinais decorrentes do envelhecimento são comumente observáveis.

\section{CONCLUSÃO}

Problemas comumente observados no processo de envelhecimento, como quedas, sedentarismo, diminuição do relacionamento social, perda da independência e autonomia, associados às doenças crônico-degenerativas, são frequentemente causados pelo declínio funcional do indivíduo que envelhece. $\mathrm{O}$ impacto financeiro desses problemas é grave, principalmente pelo aumento do contingente de idosos na população mundial. De acordo com os dados epidemiológicos observados nesta revisão bibliográfica, a porcentagem de crescimento da população idosa no Brasil era de $123 \%$ até o período em que Gosman-Hedström et al. ${ }^{11}$ conduziram o artigo Tal índice tem acarretado maior ocorrência de doenças crônico-degenerativas e suas sequelas. Assim, visando a diminuir os gastos com tratamento e manutenção da saúde da população idosa fragilizada, torna-se importante sensibilizar o sistema político brasileiro de saúde quanto à existência, finalidade e benefícios do uso da TA pelos idosos, oferecendo-lhes, desta forma, possibilidades de adquirir e utilizar os dispositivos de dispositivos de TA.

De acordo com a literatura revisada, evidências científicas concluem que o uso da TA permite ao idoso, em muitos casos, desenvolver as atividades funcionais de vida diária com segurança, aumentando sua independência e autonomia, prevenindo comorbidades e, dessa forma, contribuindo para a melhoria da sua qualidade de vida. Assim, pode-se concluir que a utilização da TA pela população idosa poderá diminuir a necessidade de cuidadores não-formais e formais, prevenir acidentes e quedas, hospitalizações e institucionalizações, além de um menor gasto financeiro com o paciente.

O profissional responsável pela avaliação, prescrição e orientação do uso dos dispositivos de TA deverá utilizar estratégias baseadas na evidência científica e clínica para propor a utilização efetiva dos mesmos junto aos indivíduos idosos fragilizados institucionalizados, hospitalizados e comunitários. Deverá também promover o envolvimento do cuidador visto que, de acordo com os estudos, ele desenvolve papel importante ao reforçar verbal e fisicamente a utilização daqueles dispositivos pelos idosos, incentivando a utilização dos mesmos. ${ }^{3}$ Para tal fazem-se necessárias orientar adequamente profissionais de saúde, bem como familiares, cuidadores e a população idosa fragilizada, sobre a existência e benefícios da TA. 
De acordo com o exposto nesta revisão bibliográfica, há um conjunto importante de artigos internacionais que retratam a utilização e os resultados do uso da TA junto à comunidade idosa fragilizada. No entanto, o número de pesquisas nacionais sobre tal tópico ainda é escasso ${ }^{7,18}$ dificultando a propagação de tais dispositivos de auxílio dentre os idosos que podem se beneficiar com os mesmos. Talvez isto seja devido ao fato de que o acesso aos recursos seja escasso e/ou a insuficiente formação de pessoal capaz de conduzir tais pesquisas. Desta forma, faz-se necessário desenvolver pesquisas nacionais sobre este tema, a fim de que se- jam verificados a necessidade e os resultados do uso de tais dispositivos junto à comunidade brasileira de idosos fragilizados e seus cuidadores.

Enfatiza-se ainda que os artigos utilizados nesta revisão bibliográfica se voltaram para idosos fragilizados, tendo sido excluídos aqueles com alterações de percepção e cognição. Portanto, seria recomendável o desenvolvimento de pesquisas brasileiras que englobassem vários tipos de fragilidade, dentre elas distúrbios de disfunções como apraxia, agnosia, afasia, demência, dentre outros.

\section{REFERÊNCIAS}

1. Papaleo Netto M. O estudo da velhice: histórico, definição do campo e termos básicos. In: Freitas EV, et al. Tratado de Geriatria e Gerontologia. Rio de Janeiro: Guanabara Koogan; 2006. p. 2-12.

2. Gitlin LN, Levine RE. Prescribing adaptive devices to the elderly: principles to treatment in the home. Int J Technol Aging 1992; 5(1): 107-20.

3. Chen T, et al. Caregiver involvement in the use of assistive devices by frail older persons. OTJR : occupation, participation and health 2002 Jul; 20(3): 179-99.

4. Gitlin LN, Luborsky MR, Schemm RL. Emerging concerns of older stroke patients about assistive device use. Gerontologist 1998 April; 38(2): 169-80.

5. Tomita M, et al. Predictors of the use of assistive device that address physical impairments among community-based frail elders. J Appl Gerontol 2004 Jun; 23(2): 141-55.
6. Freedman VA, et al. Trends in the use of assistive technology and personal care for late-life disability, 1992-2001.

Gerontologist 2006 Feb; 46(1): 124-2.

7. Mello MAF, Ramos LR. A necessidade de equipamentos de auto-ajuda e adaptações ambientais de idosos dependentes vivendo na comunidade, São Paulo, Brasil [tese]. São Paulo: UNIFESP, Escola de Medicina; 1999.

8. Agree EM, Freedman VA. Incorporating assistive devices into community-based long-term care: an analysis of the potential for substitution and supplementation. J Aging Health 2000 Aug; 12(3): 426-50.

9. Iwarsson S, Isacsson $\ddot{A}$, Lanke J. ADL dependence in the elderly population living in the community: the influence of functional limitations and physical environmental demand. Occup Ther Int 1998 Aug; 5(3): 173-93. 
10. Cornman J, Freedman V, Agree E. Measurement of assistive device use: implications for estimates of device use and disability in later life. Gerontologist 2005 Jun; 45(3): 347-58.

11. Gosman-Hedström G, et al. Use and cost of assistive technology the first year after stroke: a randomized controlled trial. Int J Technol Assess Health Care 2002 Jul; 18(3): 520-7.

12. Mann WC, et al. Effectiveness of assistive technology and environmental intervention in maintaining independence and reducing home care costs for the frail elderly: a randomized controlled trial. Arch Fam Med 1999 May; 8(3): 210-7.

13. Mann WC, et al. Frail older adults' selfreport of their most important assistive device. OTJR : occupation, participation and health 2004; 24(1): 1-9.

14. Gosman-Hedström G, Blomstrand C. Dependence and health-related quality of life in elderly people using assistive devices after acute stroke. Tecnology and Disability 2003; 15(4): 231-8.

15. Gitlin LN, Levine R, Geiger C. Adaptive device use by older adults with mixed disabilities. Arch Phys Med Rehabil 1993 Jun; 74: 149-52.

16. Agree EM, Freedman VA. A comparison of assistive technology and personal care in alleviating disability and unmet need. Gerontologist 2003 Jun; 43(3): 335-44.

17. Wessels R, et al. Non-use of provided assistive technology devices, a literature overview. Technology and disability 2003 Dec; 15(4): 231-8.

18. Mello MAF. The need of assistive devices by older dependent people living in community, São Paulo, Brazil. Proceedings of International Conference of Aging, Disability and Independence 2006, Saint Petersburg. Saint Petersburg: UFL; 2006. p.88 